\title{
Energy propagation in 1D granular soft-stiff chain
}

\author{
K. Taghizadeh ${ }^{1,2, \star}, H$. Steeb ${ }^{1}$, and S. Luding ${ }^{2}$ \\ ${ }^{1}$ Institute of Applied Mechanics (CE), SC SimTech, University of Stuttgart, Germany \\ ${ }^{2}$ Multi-Scale Mechanics, TFE, ET, MESA+, University of Twente, 7500 AE Enschede, the Netherlands
}

\begin{abstract}
When a mechanical wave travels through a medium, its intensity diminishes with distance. The research focuses on the energy transfer with distance as well as across different wavenumbers, as the mechanical wave propagates. The diffusive characteristic of energy propagation has been discussed for one-dimensional chains composed of random, pre-stressed soft and stiff particles interacting through Hertzian repulsive forces, which can be solved analytically after linearization. The effect of soft-stiff ratio (disorder in property) on energy transfer across wavenumbers is examined using a standing sinusoidal wave initial condition (with a specific wavenumber). From the total energy signals in wavenumber space, as function of time, it is observed that stronger disorder leads to more rapid loss of energy of the signal and faster transfer of energy to other wavenumbers.
\end{abstract}

\section{Introduction}

Disorder in granular matter usually occurs when mixing grains of various sizes, or different materials. Particulate mixtures are of interest for a large number of fields, materials, and applications. Complex mixtures with more than one particle species exhibit different mechanical properties than those of its ingredients. Recent studies have shown that the mechanical and acoustic behavior of binary granular mixtures are strongly influenced by the relative amount of the components [1-5].

When a mechanical wave propagates through a medium, a gradual decay of wave amplitude can be observed. In certain materials, wave pressure (amplitude) is only reduced by the spreading of the wave due to scattering [6-10]. The effect produced is to weaken the wave. Scattering is the reflection of the sound waves in directions other than its original direction of propagation.

Disorder may have an effect on the mechanical wave transmission through the granular material in its own unique way (for instance, contact disorder due to tiny polydispersity can reduce the mechanical wave speed and the transport of high frequency waves) [11-16]. Predicting the energy propagation characteristics in real and wavenumber space through disordered (simplified) model granular media, like chains, can assist in understanding the overall properties of wave propagation through real inhomogeneous media like soils.

Including disorder, e.g., adding inclusions with different properties or size, will lead to enhanced absorption in typical frequency ranges/bands, relative to the original material. The focus of this article is on the energy propagation through a 1D granular chain such that the P-wave mode is isolated from shear or rotational modes [17, 18].

^ e-mail: k.taghizadehbajgirani@utwente.nl

A video is available at https://doi.org/10.48448/nymm-h063

\section{Granular chain model}

At first, the equations of motion are derived. A 1D-chain of mesoscopic grains has been modeled using the Hertzian (nonlinear) repulsive interaction law $[19,20]$. The force between adjacent particles $i$ and $j$ ( $j$ can be $i+1$ or $i-1$ ), with mass $\tilde{m}^{(i)}$ and $\tilde{m}^{(j)}$, is:

$$
\tilde{F}_{(i, j)}=\tilde{\kappa}_{(i, j)} \tilde{\delta}_{(i, j)}^{3 / 2}, \quad \tilde{\delta}_{(i, j)} \geq 0,
$$

where $\tilde{\kappa}_{(i, j)}$ is the dimensional inter-particle contact stiffness, $\tilde{\delta}_{(i, j)}$ is the dimensional dynamic inter-particle overlap and the $3 / 2$ exponent comes from the Hertzian law. The dimensional dynamic overlap is written as $\tilde{\delta}_{(i, j)}=$ $\tilde{r}^{(i)}+\tilde{r}^{(j)}-\left|\tilde{x}^{(i)}-\tilde{x}^{(j)}\right|$ such that it is strictly non-negative for contacts, where $\tilde{r}$ and $\tilde{x}$ are the absolute dimensional radius and position, respectively. Anticipating an appropriate scaling of the problem, the tilde symbol is used for dimensional quantities. The chain has been compressed initially with a force $\tilde{P}$ such that there is some initial strain associated with the equilibrium configuration which keeps particles in contact. This assists in modeling mechanical wave propagation across well established granular chains. Assuming an external pre-compressional force $\tilde{P}$ on the granular chain in mechanical equilibrium, the initial particle overlap is given by:

$$
\tilde{\Delta}=\left(\frac{\tilde{P}}{\tilde{\kappa}_{(i, j)} \tilde{\ell}^{3 / 2}}\right)^{2 / 3}
$$

where $\tilde{\ell}$ is a length scale, i.e. characteristic lenght. Physical paramteres are scaled to obtain a non-dimensionalized equation of motion for particles. To establish a nondimensionalized equation of motion, a minimum of three number of scaling paramets are required: characteristic mass $\left(\tilde{m}_{o}\right)$ which we take as the mean particle mass of the 
system, characteristic stiffness $\left(\tilde{\kappa}_{o}\right)$ and a length scale $(\tilde{\ell})$. We choose the length scale to be related to the overlap of a characteristic contact in static equilibrium. However, one could use particle size or the driving amplitude.

To define the characteristic stiffness, we consider the contact of two identical particles of the mean mass. Under the applied compressive force, the initial overlap between such particles provides us with $\tilde{\ell}=\tilde{\Delta}_{0}$ (with nondimensional initial overlap $\left.\Delta_{0}=\tilde{\Delta}_{0} / \tilde{\ell}=1\right)$. Inserting the scaled particle overlap $\delta_{(i, j)}=\tilde{\delta}_{(i, j)} / \tilde{\ell}$ in Eq. (1) yields:

$$
\tilde{F}_{(i, j)}=\tilde{\kappa}_{(i, j)} \tilde{\ell}^{3 / 2} \delta_{(i, j)}^{3 / 2},
$$

The non-dimensional mass $b^{(i)}=\tilde{m}^{(i)} / \tilde{m}_{o}$, the nondimensional stiffness is $\kappa_{(i, j)}=\tilde{\kappa}_{(i, j)} / \tilde{\kappa}_{o}$ and the nondimensional displacement is $u=\tilde{u} / \tilde{\ell}$. Without new scaling parameters, this also defines the non-dimensional time $t=\tilde{t} / \tilde{t}_{c}$ where

$$
\tilde{t}_{c}=\sqrt{\frac{\tilde{m}_{o}}{\tilde{\kappa}_{o} \tilde{\ell}^{1 / 2}}},
$$

thus, the non-dimensional repulsive interaction force becomes:

$$
F_{(i, j)}=\frac{\tilde{t}_{c}^{2}}{\tilde{m}_{o} \tilde{\ell}} \tilde{F}_{(i, j)}
$$

We write an equation of motion for the general particle $i(i=1, \ldots, \mathrm{N})$ as:

$$
\tilde{m}^{(i)} \frac{\mathrm{d}^{2} \tilde{x}^{(i)}}{\mathrm{d} \tilde{t}^{2}}=\tilde{\kappa}_{(i-1, i)} \tilde{\ell}^{3 / 2} \delta_{(i-1, i)}^{3 / 2}-\tilde{\kappa}_{(i, i+1)} \tilde{\ell}^{3 / 2} \delta_{(i, i+1)}^{3 / 2}
$$

We denote the displacement of particle $i$ from its equilibrium position $\tilde{x}_{0}^{(i)}$ as $\tilde{u}^{(i)}=\tilde{\ell} u^{(i)}=\tilde{x}^{(i)}-\tilde{x}_{0}^{(i)}$. Thus, for a contact between $i$ and $j$ (with $j>i$ ), the scaled overlap is $\delta_{(i, j)}=\Delta+u^{(i)}-u^{(j)}$.

The non-dimensional equation of motion for particle $i$ is now given by

$$
\begin{aligned}
b^{(i)} \frac{\mathrm{d}^{2} u^{(i)}}{\mathrm{d} t^{2}} & =F_{(i-1, i)}-F_{(i, i+1)} \\
& =\kappa_{(i-1, i)}\left[\Delta_{(i-1, i)}-\left(u^{(i)}-u^{(i-1)}\right)\right]^{3 / 2} \\
& -\kappa_{(i, i+1)}\left[\Delta_{(i, i+1)}-\left(u^{(i+1)}-u^{(i)}\right)\right]^{3 / 2},
\end{aligned}
$$

where the stiffness ratio $\kappa_{(i, j)}=\kappa_{(i, j)} / \tilde{\kappa_{0}}$ has been defined implicitly. Note that in this study, $\kappa_{(i, j)}$ is the disorder parameter. The implication of the disorder is explained later.

\subsection{Linearized equation of motion}

Here, we linearize the general force-displacement relation about the equilibrium configuration. The nondimensional phrasing of Eq. (1) is given by:

$$
F_{(i, j)}=\kappa_{(i, j)} \delta_{(i, j)}^{3 / 2}
$$

which can be expanded around the initial overlap $\Delta_{(i, j)}$, if the amplitudes of the displacement $u^{(i)}$ are small during mechanical wave propagation, so the relative displacements $\delta_{(i, j)}-\Delta_{(i, j)}=u^{(i)}-u^{(j)}$, and the nonlinear terms can be ignored so that:

$$
F_{(i, j)} \cong \kappa_{(i, j)} \Delta_{(i, j)}^{3 / 2}-\frac{3}{2} \kappa_{(i, j)} \Delta_{(i, j)}^{1 / 2}\left(u^{(j)}-u^{(i)}\right) .
$$

There are $p=0, \ldots, N+1$ (in total $N+2$ ) particles in the granular chain with particles 0 and $(N+1)$ as the boundaries of the chain such that $u^{(0)}=0$ and $u^{(N+1)}=$ 0 . Alternatively, periodic boundaries are realized by using $N+1$ particles, equivalent to setting $u^{(0)}=u^{(N+1)} \neq 0$, that is also allowed to move, so all vectors and matrices get length $N+1$ with $2^{i}=N+1$, with $i$ integer. Eq. (9) results in $N$ equations which are assembled in a matrix form:

$$
\mathbf{M} \frac{\mathrm{d}^{2} \mathbf{u}}{\mathrm{d} t^{2}}=\mathbf{K u}
$$

where $\mathbf{M}$ is a diagonal matrix with $b^{(1)}, b^{(2)}, b^{(3)} \ldots . b^{(N)}$ as diagonal elements, $\mathbf{K}$ is a symmetric, tri-diagonal matrix with $-\frac{3}{2}\left(\kappa_{(i+1, i)}^{2 / 3}+\kappa_{(i-1, i)}^{2 / 3}\right)$ as diagonal, $\frac{3}{2} \kappa_{(i+1, i)}^{2 / 3}$ as superdiagonal and $\frac{3}{2} \kappa_{(i-1, i)}^{2 / 3}$ as subdiagonal elements, other elements of $\mathbf{K}$ are $0 . \mathbf{u}$ is the displacement vector containing displacements $u^{(i)}$ as elements. The solution of this equation is given in eigenspace and real space, respectively, by:

$$
\mathbf{z}(t)=\mathbf{C}^{(1)} \mathbf{a}+\mathbf{C}^{(2)} \mathbf{b} \quad \text { or } \quad \mathbf{u}(t)=\mathbf{S} \mathbf{C}^{(1)} \mathbf{a}+\mathbf{S C}^{(2)} \mathbf{b},
$$

where $\mathbf{C}^{(1)}$ is a diagonal matrix with $\sin \left(\omega_{j} t\right)$ as diagonal elements, $\mathbf{C}^{(2)}$ is also a diagonal matrix with $\cos \left(\omega_{j} t\right)$ as diagonal elements, $\mathbf{a}$ and $\mathbf{b}$ are vectors which are determined from initial conditions $\mathbf{u}_{o}$ (initial displacement vector) and $\mathbf{v}_{o}$ (initial velocity vector).

$$
\mathbf{a}=\mathbf{H}^{-1} \mathbf{S}^{-1} \mathbf{v}_{o} \quad \text { and } \quad \mathbf{b}=\mathbf{S}^{-1} \mathbf{u}_{o},
$$

where $\mathbf{H}$ is a diagonal matrix with $\omega_{j}$ as the diagonal ele-

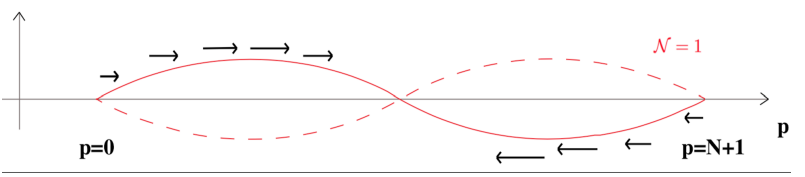

Figure 1. Sinusoidal waveform initial condition for a fixed-end and periodic granular chain.

\subsection{Standing wave condition}

An initial sinusoidal waveform (see Fig. 1) is applied to the (periodic) chain in the form of $\mathbf{u}_{o}=u_{o} \sin \left(\mathcal{N} \frac{2 \pi p}{N+1}\right)$ and $\mathbf{v}_{o}=0$ (where $p=1, \ldots, N+1$ or $0, \ldots, N, \mathcal{N}=1, \ldots$, $(N+1) / 2$ specifies the particular tone of the standing wave). It is important to avoid opening and closing of contacts by setting the condition $u_{o} \ll 1$ (initial particle overlap $\Delta_{o}$ ) in order to hold the validity of the linearized equations of motion (Sect. 2.1). $\mathbf{a}$ and $\mathbf{b}$ are given as:

$$
\mathbf{a}=\mathbf{H}^{-1} \mathbf{S}^{-1} \mathbf{v}_{o}=0 \text { and } \mathbf{b}=\mathbf{S}^{-1} \mathbf{u}_{o} .
$$

Hence, the displacement and velocity of the particles become:

$$
\mathbf{u}=\mathbf{S} \mathbf{C}^{(2)} \mathbf{S}^{-1} \mathbf{u}_{o} \quad \text { and } \quad \mathbf{v}=-\mathbf{S H C} \mathbf{C}^{(1)} \mathbf{S}^{-1} \mathbf{u}_{o} ;
$$

which is also written as:

$$
\begin{aligned}
& u^{(i)}(t)=u_{0} \sum_{j=1}^{N} S_{i j} \cos \left(\omega_{j} t\right) \sum_{p=1}^{N} S_{p j} \sin \left(\mathcal{N} \frac{2 \pi i}{N+1}\right), \\
& v^{(i)}(t)=-u_{0} \sum_{j=1}^{N} \omega_{j} S_{i j} \sin \left(\omega_{j} t\right) \sum_{p=1}^{N} S_{p j} \sin \left(\mathcal{N} \frac{2 \pi i}{N+1}\right) .
\end{aligned}
$$




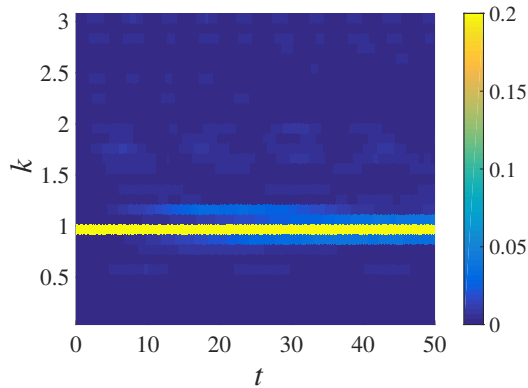

(a)

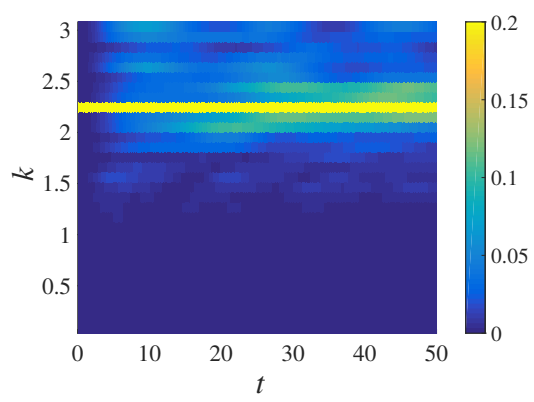

(d)

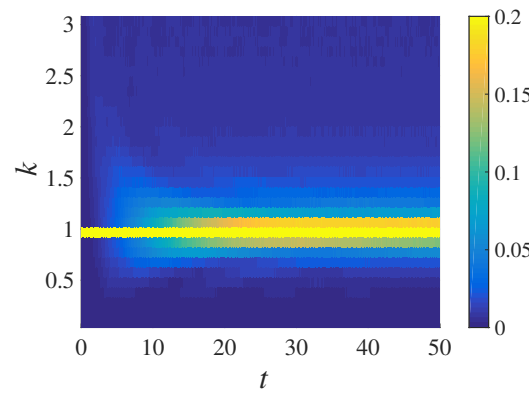

(b)

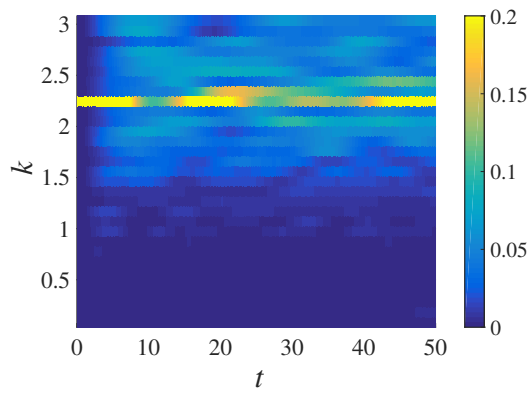

(e)

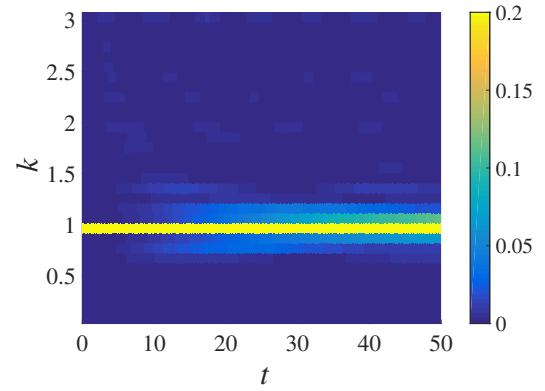

(c)

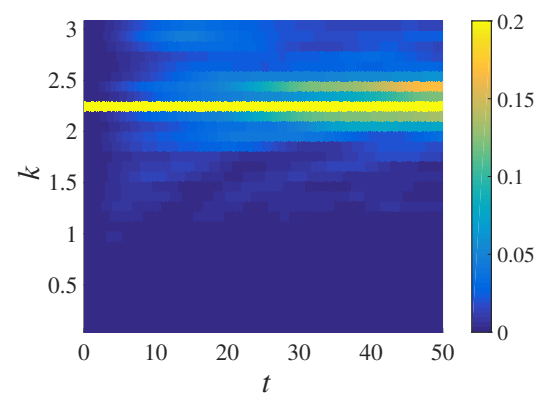

(f)

Figure 2. Binned total energy response $(\langle\hat{e}(t)\rangle)$ of chains made with ratio $v=0.1(\mathrm{a}, \mathrm{d}), 0.5(\mathrm{~b}, \mathrm{e})$, and 0.9 (c,f) agitated initially with $k_{\text {ins }}=0.981$ (top row) and $k_{\text {ins }}=2.159$ (bottom row).

\subsection{Total energy and binning energy}

In this section, we define the total energy calculated in wavenumber space. The Total Energy $\mathbf{E}_{\text {tot }}$ of individual particles is the sum of its kinetic $\mathbf{E}_{\mathbf{k i n}}$ and potential energies $\mathbf{E}_{\text {pot }}$ :

$$
\mathbf{E}_{\text {tot }}=\mathbf{E}_{\text {kin }}+\mathbf{E}_{\text {pot }} \text {. }
$$

Using expressions obtained earlier, Eq. (15), the total energy is written as:

$$
E_{\text {tot }}^{(p)}(t)=\frac{1}{2}(b(p))\left(v^{(p)}(t)\right)^{2}-\frac{1}{2} F^{(p)}(t) u^{(p)}(t) .
$$

After that, the total energy calculated in wavenumber space is binned. This helps to deal with one eigenmode per a group with an averaged total energy instead of many eignemodes. If we consider $\Delta k$ the bandwidth of the bin and $r$ the central wavenumber, the binning of the total energy becomes:

$$
e^{(r)}(t)=\sum_{r-\Delta k / 2}^{r+\Delta k / 2} E_{t o t}^{(k)}(t)
$$

Since the total energy is conserved, hence:

$$
\sum_{r=1}^{B} e^{(r)}(t)=\sum_{k} E_{t o t}^{(k)}(t),
$$

where $B$ is the total number of bins assigned in wavenumber space. The energy results shown later are normalized by the total energy as a probability density:

$$
\hat{e}^{(r)}(t)=\frac{e^{(r)}(t)}{\sum_{r=1}^{B} e^{(r)}(t)} .
$$

\subsection{Implication of disorder in a monodisperse chain}

In earlier studies, the effect of mass and inter-particle contact stiffness disorder in a pre-stressed monodisperce granular chain subjected to ballistic and harmonic perturbation of the boundary was studied [13, 21-23].

In the current study, two different types of particles, so called soft and stiff, are chosen for creation of a chain. The mass and inter-particle stiffness between the soft particles are assigned to be half of the mass and inter-particle stiffness of the stiff particles. Also, the inter-particle stiffness between soft and stiff particles are set to be $2 / 3$ of the stiff particles. Particles are placed randomly in the granular chain at every realization.

\section{Results and discussion}

$N$ particles long granular chains with three different soft to stiff ratios (number of soft to stiff particles in chain), $v=0.1,0.5$, and 0.9 , have been used with standing wave initial conditions. This section deals with the analysis energy transfer between different wave numbers in time. In order to improve the quality of the signals, every content ratio analysis was averaged over 100 realizations.

Eq. (17) is used to obtain total energy in wavenumber space, then Eq. (20) is used to bin the energy responses accordingly. The number of bins used for the computation here is $B=32$ with a bandwidth $\Delta k=\pi / 32=0.0982$. Fig. 2 shows the evolution of the binned total energy in wave number space (color scale in the plots is $E_{t o t}^{(k)}(t)$ ) for bands initially agitated at wave number, $k_{\text {ins }}=\mathcal{N} \frac{2 \pi}{N+1}=$ 0.981 (top plots) and 2.159 (bottom plots) which correspond to bin numbers $r=10$ and $r=23$. 
A peak is initially observed at the agitated wavenumber $\left(k_{i n s}\right)$, the peak decreases as the time progresses, the decay rate is lower for lower wavenumber and lesser energy transferred to other bands, which can be observed when top plots are compared with the bottom ones.

Comparing the plots for $v=0.5$ with others, it is found that higher disorder in a chain leads to faster energy transfer to other bands. In other words, when the disorder of the chain is higher regardless of the stiffness of particles, the attenuation takes place faster with incorporation of more neighboring bands.

\section{Conclusion}

In this study, the influence of soft-stiff ratio disorder on energy propagation was examined using a harmonic wave propagation in a granular chain, where soft and stiff particles are distributed randomly in the chain.

At first, the micro-mechanical model of the granular chain with linearized Hertzian repulsive interaction forces acting between the granules was explained. After that, the equations used for computing the total energy response in wavenumber space. Calculated total energy were grouped in order to deal with fewer wavenumbers. From the results it can be concluded that higher disorder causes a faster decay of higher wavenumbers, whereas lower wavenumbers remain in the system for a longer time.

In future work, the diffusive characteristic of energy propagation and its frequency dependence will be included into a reduced order model to establish a master equation with the aid of a one-dimensional soft-stiff disordered granular chain.

\section{Acknowledgement}

Authors acknowledge funding from the German Science Foundation (DFG) through the project STE-969/16-1 within the SPP 1897 "Calm, Smooth and Smart".

\section{References}

[1] J.S. Lee, J. Dodds, J.C. Santamarina, J. Mater. Civil Eng. 19, 179-184 (2007)

[2] J.R. Valdes, T.M Evans, Can. Geotech. J. 45, 588-595 (2008)

[3] H.K. Kim, J.C. Santamarina, Can. Geotech. J. 45, 1457-1466 (2008)
[4] K. Taghizadeh, H. Steeb, V. Magnanimo, S. Luding, EPJ Web of Conferences 140, 12019 (2017)

[5] K. Taghizadeh, S. Luding, V. Magnanimo, ALERT Doctoral School 2017 Discrete Element Modeling p.129 (2017)

[6] K. Aki, B. Chouet, J.Geophys.Res. 80, 3322-3342 (1975)

[7] R.L. Weaver, W. Sachse, J. Acoust. Soc. Am. 97, 2094-2102 (1995)

[8] I. Güven, S. Luding, H. Steeb, J. Vib. Acoust. 140, 011018 (2018)

[9] J.H. Page, Nano Optics and Atomics: Transport of Light and Matter Waves (IOS Press, Amsterdam, 2011)

[10] L. Trujillo, F. Peniche, X. Jia, Multiple scattering of elastic waves in granular media: Theory and experiments (INTECH Open Access Publisher, 2011)

[11] O. Mouraille, S. Luding, Ultrasonics 48, 498-505 (2008)

[12] C. Coste, B. Gilles, Phys. Rev. E 77, 021302 (2008)

[13] B.P. Lawney, S. Luding, AIP Conference Proceedings 1542, 535-538 (2013)

[14] O. Mouraille, S. Luding, Partec 2007, (2007)

[15] J.A. Scales, E.V. Vleck, S. Erik, J.Comput.Phys. 133, 27-42 (1997)

[16] Q. Zhang, W. Li, J. Lambros, L.A. Bergman, A.F. Vakakis, Granul. Matter 22, 1-16 (2020)

[17] J. Yang, M. Gonzalez, E. Kim, C. Agbasi, M. Sutton, Exp. Mech. 54, 1043-1057 (2014)

[18] A. Misra, N. Nejadsadeghi, Wave Motion 90, 175195 (2019)

[19] L.D. Landau, E.M. Lifshitz, Theory of elasticity (Pergamon Press, Oxford, 2011)

[20] C.F. Schreck, C.S. O'Hern, M.D. Shattuck, Granul. Matter 16, 209-216 (2014)

[21] O.J.P. Mouraille, Ph.D thesis, Sound propagation in dry granular materials: discrete element simulations, theory, and experiments (University of Twente, Netherlands, 2009)

[22] K. Taghizadeh, Ph.D thesis, Elasticity and wave propagation in granular materials (University of Twente, Netherlands, 2019)

[23] R.K. Shrivastava, Ph.D thesis, Towards Stochastic and Deterministic Modeling of Mechanical Waves in Disordered Media (University of Twente, Netherlands, 2018) 